\title{
NOTE ON THE GEOMETRIC INTERPRETATION OF THE VANISHING OF A CERTAIN PROJECTIVE INVARIANT OF TWO CONICS*
}

\section{ROBIN ROBINSON}

1. Introduction. The envelope of a line moving so that its pairs of intersections with two conics form a harmonic set is a third conic; the eight tangents to the given conics at their four points of intersection touch this envelope. The discriminant of this envelope is a relative invariant of weight four of the pair of ternary quadratic forms defining the given conic, and is the projective invariant mentioned in the title. When it vanishes, the envelope is degenerate, and the eight tangents mentioned above pass by fours through two points. $\dagger$

Let the point-equations of the given conics be

$$
\sum a_{i j} x_{i} x_{j}=0, \quad a_{i j}=a_{j i}, \quad \sum b_{i j} x_{i} x_{j}=0, \quad b_{i j}=b_{j i} .
$$

Then their line-equations are

$$
\sum A_{i j} u_{i} u_{j}=0, \quad \sum B_{i j} u_{i} u_{j}=0,
$$

where $A_{i j}$ is the cofactor of $a_{i j}$ in the determinant $\left|a_{i j}\right|$, and similarly for $B_{i j}$. The line-equation of the envelope is then

$$
\sum \alpha_{i j} u_{i} u_{j}=0,
$$

where $\alpha_{i j}$ is found by the Aronhold process: $\ddagger$

$$
\alpha_{i j} \equiv \sum \frac{\partial A_{i j}}{\partial a_{k l}} b_{k l} \equiv \sum a_{k l} \frac{\partial B_{i j}}{\partial b_{k l}} .
$$

The condition that the envelope be degenerate is then $\left|\alpha_{i j}\right|=0$. For want of a better term, we shall say that the two given conics are related if the envelope is degenerate. We shall proceed to prove a number of properties of related conics. Although most

* Presented to the Society, December 27, 1934.

† Salmon, Conic Sections, 1917, pp. 306 ff., 345; Clebsch-Lindemann, Vorlesungen über Geometrie, 1932, vol. 1, pt. 1, pp. 513, 523.

$\ddagger$ Clebsch-Lindemann, loc. cit., pp. 501, 513 . 
of these properties are more general, we shall limit our discussion to related conics intersecting in four distinct points.

2. Related Conics. The locus of a point moving so that the lines joining it to the four points $A_{1}(1,0,0), A_{2}(0,1,0)$, $A_{3}(0,0,1), A_{4}(1,1,1)$, respectively, have the cross-ratio $\lambda$ is the conic

$$
(1-\lambda) x_{1} x_{2}-x_{2} x_{3}+\lambda x_{3} x_{1}=0 .
$$

Here $\lambda$ represents not only the cross-ratio of the four lines, but also of the four points as points of the second order pencil lying on the conic. As $\lambda$ varies we obtain a whole pencil of conics, the degenerate cases being given by $\lambda=0,1, \infty$. Let us determine under what conditions two non-degenerate conics of the pencil are related. The equation in line-coordinates of the conic $C$ given by $\lambda$ is readily found to be

$$
\begin{aligned}
& u_{1}^{2}+\lambda^{2} u_{2}^{2}+(1-\lambda)^{2} u_{3}^{2}+2 \lambda u_{1} u_{2}-2 \lambda(1-\lambda) u_{2} u_{3} \\
& \quad+2(1-\lambda) u_{3} u_{1}=0 .
\end{aligned}
$$

If these coefficients are now polarized by the Aronhold process with respect to the coefficients of a second conic of the pencil, $C^{\prime}$, given by $\lambda^{\prime}$, the resulting coefficients $\alpha_{i j}$ are those for the envelope, whose equation is thus

$$
\begin{aligned}
& u_{1}^{2}+\lambda \lambda^{\prime} u_{2}^{2}+(1-\lambda)\left(1-\lambda^{\prime}\right) u_{3}^{2}+\left(\lambda+\lambda^{\prime}\right) u_{1} u_{2} \\
& \quad+\left[2 \lambda \lambda^{\prime}-\left(\lambda+\lambda^{\prime}\right)\right] u_{2} u_{3}+\left[2-\left(\lambda+\lambda^{\prime}\right)\right] u_{3} u_{1}=0 .
\end{aligned}
$$

Its discriminant is

$$
\left|\alpha_{i j}\right|=4\left(\lambda+\lambda^{\prime}\right)\left[2-\left(\lambda+\lambda^{\prime}\right)\right]\left[2 \lambda \lambda^{\prime}-\left(\lambda+\lambda^{\prime}\right)\right]
$$

and the condition that the two given conics be related is $\left|\alpha_{i j}\right|=0$, that is, $\lambda+\lambda^{\prime}=0$, or $\lambda+\lambda^{\prime}=2$, or $1 / \lambda+1 / \lambda^{\prime}=2$. These relations can also be written

$\lambda^{\prime}=-\lambda, \quad 1-\lambda^{\prime}=-(1-\lambda), \quad 1-1 / \lambda^{\prime}=-(1-1 / \lambda)$.

Since $\lambda, 1-\lambda$, and $1-1 / \lambda$ and their reciprocals are the six crossratios of the four base-points in different orders, we may state the following theorem.

TheOREM 1. A necessary and sufficient condition that two nondegenerate conics with four distinct intersections be related is that 
the cross-ratio of these four intersections in some order as points of one conic be the negative of the corresponding cross-ratio for the other conic.

The proper order of the intersections gives us a pairing of what we shall call associated intersections. For example, if $\lambda^{\prime}=-\lambda$, since $\lambda=\left(A_{1} A_{2}, A_{3} A_{4}\right), A_{1}$ and $A_{2}$ are associated intersections, as also are $A_{3}$ and $A_{4}$.

If the above three relations are written $\lambda^{\prime}=-\lambda, \lambda^{\prime}=2-\lambda$, $\lambda^{\prime}=\lambda /(2 \lambda-1)$, the values $\lambda=-1,2,1 / 2$ are each seen to make one of the values of $\lambda^{\prime}$ correspond to a degenerate conic, and hence we have the following theorem.

THEOREM 2. In a pencil of conics with four distinct base-points, there are just three non-degenerate conics which are related to a given non-degenerate conic, unless the base-points form a harmonic set on the given conic, when there are just two non-degenerate related conics. One of these related conics corresponds to each pairing of the four base-points, except when the two pairs separate one another harmonically.

Let us now consider the tangents to two distinct non-degenerate conics $C(\lambda)$ and $C^{\prime}\left(\lambda^{\prime}\right)$ at a common point, say $A_{1}(1,0,0)$. They are respectively

$$
T_{1}:(1-\lambda) x_{2}+\lambda x_{3}=0, \quad T_{1}^{\prime}:\left(1-\lambda^{\prime}\right) x_{2}+\lambda^{\prime} x_{3}=0 .
$$

Solving simultaneously the equations of $T_{1}$ and

$$
C^{\prime}:\left(1-\lambda^{\prime}\right) x_{1} x_{2}-x_{2} x_{3}+\lambda^{\prime} x_{3} x_{1}=0,
$$

we find that they intersect at $A_{1}$ and

$$
S_{1}:\left(1, \frac{\lambda^{\prime}-\lambda}{1-\lambda},-\frac{\lambda^{\prime}-\lambda}{\lambda}\right) .
$$

Similarly, $T_{1}^{\prime}$ meets $C$ at $A_{1}$ and

$$
S_{1}^{\prime}:\left(1,-\frac{\lambda^{\prime}-\lambda}{1-\lambda^{\prime}}, \frac{\lambda^{\prime}-\lambda}{\lambda^{\prime}}\right) .
$$

For convenience we shall call $S_{1}$ and $S_{1}^{\prime}$ the satellite points of $A_{1}$.

Now $S_{1}$ and $S_{1}^{\prime}$ are collinear with $A_{2}(0,1,0)$ if and only if the determinant of the three sets of coordinates vanishes, that 
is, if and only if $\lambda^{\prime}=-\lambda$, in other words, if and only if $C$ and $C^{\prime}$ are related conics with $A_{1}$ and $A_{2}$ associated intersections.

Similarly, $S_{1}$ and $S_{1}{ }^{\prime}$ are collinear with $A_{3}(0,0,1)$ if and only if $\lambda^{\prime}=2-\lambda$, and with $A_{4}(1,1,1)$ if and only if $\lambda^{\prime}=\lambda /(2 \lambda-1)$.

It is clear from this that if $\lambda^{\prime}=-\lambda$, the satellite points of $A_{2}$ are collinear with $A_{1}$, those of $A_{3}$ with $A_{4}$, and those of $A_{4}$ with $A_{3}$. Hence we may state the following theorem.

TheOREM 3. A necessary and sufficient condition that two nondegenerate conics with four distinct intersections be related is that the satellite points of a point of intersection be collinear with a second point of intersection; when this is true, the satellite points of each intersection are collinear with the associated intersection.

It is interesting to note that if the two conics are circles, then a necessary and sufficient condition that they be related with their finite intersections associated is that they be orthogonal.* This property suggests related conics as a natural projective generalization of orthogonal circles, which indeed they are.

That related conics are not a natural generalization of orthogonal conics in general is readily illustrated by the case of the ellipse $b_{1}^{2} x^{2}+a_{1}^{2} y^{2}=a_{1}^{2} b_{1}^{2}$ and the hyperbola $b_{2}^{2} x^{2}-a_{2}^{2} y^{2}=a_{2}^{2} b_{2}^{2}$, which are orthogonal if and only if confocal. The writer has found that they are related with their first and second quadrant intersections associated if and only if $b_{1}=b_{2}$.

Since in the case of related conics the envelope mentioned in the first paragraph is degenerate, the eight tangents $T_{i}, T_{i}{ }^{\prime}$, $(i=1,2,3,4)$, to $C$ and $C^{\prime}$ at $A_{i},(i=1,2,3,4)$, pass by fours through two points. In case $\lambda^{\prime}=-\lambda, T_{1}, T_{2}, T_{3}^{\prime}, T_{4}^{\prime}$ are concurrent at the point $(1,-\lambda, 1-\lambda)$, while $T_{3}, T_{4}, T_{1}^{\prime}, T_{2}^{\prime}$ are concurrent at $(1, \lambda, 1+\lambda)$. The two sets are perspective in the line $x_{1}-x_{2}+x_{3}=0$ (and also in another order in the line $x_{1}-x_{2}-x_{3}$ $=0$ ), and hence have the same cross-ratio. From the equations

$$
\begin{aligned}
T_{1}: & (1-\lambda) x_{2}+\lambda x_{3}=0, & T_{2}: & (1-\lambda) x_{1}-x_{3}=0, \\
T_{3}^{\prime}: & \lambda x_{1}+x_{2}=0, & T_{4}^{\prime}: & x_{1}+\lambda x_{2}-(1+\lambda) x_{3}=0,
\end{aligned}
$$

we see that $T_{3}^{\prime} \sim T_{1}+\lambda T_{2}, T_{4}^{\prime} \sim \lambda T_{1}+T_{2}$, so that the cross-ratio is $\lambda^{2}$.

\footnotetext{
* Salmon, loc. cit., pp. 348 ff.
} 
THEOREM 4. If two conics are related, the eight tangents to them at their four points of intersection are concurrent in two perspective sets of four each, one of whose common cross-ratios is the square of the corresponding cross-ratio of the four corresponding intersections regarded as points of one of the conics.

In case the two conics $C$ and $C^{\prime}$ are related with $A_{1}$ and $A_{2}$ as associated intersections, $\lambda^{\prime}=-\lambda$, and the satellite points of $A_{1}$ become

$$
S_{1}:\left(1, \frac{-2 \lambda}{1-\lambda}, 2\right), \quad S_{1}^{\prime}:\left(1, \frac{2 \lambda}{1+\lambda}, 2\right),
$$

and their join becomes the line $x_{3}=2 x_{1}$. Since this is independent of $\lambda$, we have the following theorem.

THEOREM 5. If each non-degenerate conic of a pencil with four distinct base-points is paired with the related conic for which two definite base-points are associated intersections, the satellite points of one intersection all lie on the same straight line through the other intersection.

3. Related Line Conics. It is of course desirable to consider the dual of two related conics. The locus of a point moving so that the four tangents drawn from it to two given conics form a harmonic set is a third conic, and the two given conics are related in the dual sense if this locus is degenerate. Suffice it to say that for the conics $C$ and $C^{\prime}$ this locus has the equation

$$
\begin{aligned}
& \left(\lambda-\lambda^{\prime}\right)^{2}\left(x_{1}^{2}+x_{2}^{2}+x_{3}^{2}\right)+2\left[2-\left(\lambda+\lambda^{\prime}\right)\right]\left[2 \lambda \lambda^{\prime}-\left(\lambda+\lambda^{\prime}\right)\right] x_{1} x_{2} \\
& \quad+2\left(\lambda+\lambda^{\prime}\right)\left[2-\left(\lambda+\lambda^{\prime}\right)\right] x_{2} x_{3} \\
& \quad+2\left(\lambda+\lambda^{\prime}\right)\left[2 \lambda \lambda^{\prime}-\left(\lambda+\lambda^{\prime}\right)\right] x_{3} x_{1}=0,
\end{aligned}
$$

and that its discriminant is

$$
-16 \lambda \lambda^{\prime}(1-\lambda)\left(1-\lambda^{\prime}\right)\left(\lambda+\lambda^{\prime}\right)\left[2-\left(\lambda+\lambda^{\prime}\right)\right]\left[2 \lambda \lambda^{\prime}-\left(\lambda+\lambda^{\prime}\right)\right] \text {. }
$$

Since neither $C$ nor $C^{\prime}$ is degenerate, $\lambda \lambda^{\prime}(1-\lambda)\left(1-\lambda^{\prime}\right) \neq 0$, and hence this locus is degenerate when and only when $C$ and $C^{\prime}$ are related in the original sense. Hence we have the following theorems.

TheOREM 6. Relatedness is a self-dual property.

THEOREM 7. Two non-degenerate conics with four distinct com- 
mon tangents are related if and only if the cross-ratio of these four common tangents in some order as lines of one conic is the negative of the corresponding cross-ratio for the other conic.

If the other tangents to $C^{\prime}$ and $C$ drawn from the points of contact of a common tangent with $C$ and $C^{\prime}$, respectively, be termed satellite lines of the common tangent, then we have the following theorem.

THEOREM 8. The satellite lines of a common tangent to two nondegenerate conics are concurrent with another common tangent if and only if the conics are related.

COROLlaRY. The satellite lines of a real common tangent to two orthogonal circles are concurrent with the other real common tangent.

4. Osculating Cubic of Related Conics. In order to study the next property of related conics we shall introduce a non-homogeneous coordinate system in which $A_{1}$ is the origin, $A_{1} S_{1}$ and $A_{1} S_{1}^{\prime}$ the $x$ and $y$ axes, respectively, and $S_{1} S_{1}^{\prime}$ the line at infinity. Since then $C$ is tangent to the $x$ axis at the origin and passes through the point at infinity on the $y$ axis, its equation is

$$
C: \quad x^{2}-a x y-b y=0, \text { or } y=x^{2} /(a x+b) .
$$

Similarly,

$$
C^{\prime}:-c x y+y^{2}-d x=0, \text { or } x=y^{2} /(c x+d) .
$$

Any cubic having a node at the origin with the axes as nodal tangents will have an equation of the form

$$
Q_{1}: \quad \alpha x^{3}+\beta x^{2} y+\gamma x y^{2}+\delta y^{3}-b d x y=0 .
$$

It is clear that the undetermined coefficients $\alpha, \beta, \gamma, \delta$ leave us just enough freedom to make $Q_{1}$ have four-point contact with each of $C$ and $C^{\prime}$ at $A_{1}$. For example, if $Q_{1}$ and $C$ are to have fourpoint contact at $A_{1}$, they must intersect five times there (four times for one branch of $Q_{1}$, once for the other). That is, if we substitute $y=x^{2} /(a x+b)$ in the left member of the equation of $Q_{1}, x^{5}$ must be a factor of the resulting expression. Substituting and multiplying by $(a x+b)^{3}$, we obtain the equation

$$
\begin{aligned}
& \alpha x^{3}(a x+b)^{3}+\beta x^{4}(a x+b)^{2}+\gamma x^{5}(a x+b) \\
& \quad+\delta x^{6}-b d x^{3}(a x+b)^{2}=0 .
\end{aligned}
$$


Equating to zero the coefficients of $x^{3}$ and $x^{4}$, we have

$$
\begin{aligned}
\alpha b^{3}-b^{3} d & =0, & \alpha & =d, \\
3 \alpha a b^{2}+\beta b^{2}-2 a b^{2} d & =0, & \beta & =-a d .
\end{aligned}
$$

Performing the same process with $Q_{1}$ and $C^{\prime}$, we obtain the relations $\gamma=-b c, \delta=b$. Hence the equation of $Q_{1}$ becomes

$$
d x^{3}-a d x^{2} y-b c x y^{2}+b y^{3}-b d x y=0 .
$$

This we shall call the osculating cubic of $C$ and $C^{\prime}$ for the intersection $A_{1}$. Since $Q_{1}$ cuts each of $C$ and $C^{\prime}$ five times at $A_{1}$, it must have a single intersection with each somewhere else. If we multiply the first equation given above for $C$ by $d x$ we obtain $d x^{3}-a d x^{2} y-b d x y=0$. Subtracting from the equation of $Q_{1}$, we have $-b c x y^{2}+b y^{3}=0$, and so the remaining intersection lies on the line $y=c x$, which also passes through the intersection of $C^{\prime}$ with the line at infinity other than $S_{1}$. In a similar manner, the remaining intersection of $Q_{1}$ and $C^{\prime}$ lies on $x=a y$, which also passes through the intersection of $C$ with the line at infinity other than $S_{1}^{\prime}$.

Now a necessary and sufficient condition that $C$ and $C^{\prime}$ be related is that these extra intersections with the line at infinity be coincident, that is, $a c=1$, in which case they also coincide with the extra points common to $Q_{1}$ and $C$, and to $Q_{1}$ and $C^{\prime}$; and conversely. Hence we have the following theorem.

TheOREM 9. A necessary and sufficient condition that two nondegenerate conics be related is that the osculating cubic at any intersection pass through another intersection, which in this case is the associated intersection.

Curiously enough, it was this property in the case of two orthogonal circles which introduced the writer to this problem.

Returning now to our original coordinate system, and to the related conics $C$ and $C^{\prime}$ for which $\lambda^{\prime}=-\lambda$, I remark that it is merely a matter of detail which I shall spare the reader to show that the equation of the osculating cubic $Q_{1}$ at $A_{1}$ is

$$
Q_{1}: \quad\left(3 x_{3}-2 x_{1}\right) x_{2}^{2}+\lambda^{2}\left(x_{3}+2 x_{1}\right)\left(x_{2}-x_{3}\right)^{2}=0 .
$$

As $\lambda$ changes, the cubic $Q_{1}$ for the pairs of conics $C$ and $C^{\prime}$ form a pencil, with base-points as follows: 
Quadruple base-point: $A_{1}(1,0,0)$, common node;

Double base-points: $(1,0,-2)$, common tangent $x_{3}+2 x_{1}=0$; $(3,2,2)$, common tangent $3 x_{3}-2 x_{1}=0$;

Simple base-point: $\quad A_{2}(0,1,0)$, associated intersection. It is interesting to note that the common tangents at both double base-points pass through $A_{2}$. Moreover, $x_{3}+2 x_{1}=0$ is the harmonic conjugate of $S_{1} S_{1}^{\prime} \quad\left(x_{3}=2 x_{1}\right)$ with respect to the lines $A_{1} A_{2}\left(x_{3}=0\right)$ and $A_{2} A_{3}\left(x_{1}=0\right)$, while $3 x_{3}-2 x_{1}=0$ is the harmonic conjugate of $S_{1} S_{1}^{\prime}\left(x_{3}=2 x_{1}\right)$ with respect to $A_{1} A_{2}\left(x_{3}=0\right)$ and $A_{2} A_{4}\left(x_{3}=x_{1}\right)$.

By applying the involution

$$
\rho x_{1}^{\prime}=x_{3}-x_{2}, \quad \rho x_{2}^{\prime}=x_{3}-x_{1}, \quad \rho x_{3}^{\prime}=x_{3},
$$

which interchanges $A_{1}$ and $A_{2}, A_{3}$ and $A_{4}$, and leaves $C$ and $C^{\prime}$ invariant, $Q_{1}$ is transformed into $Q_{2}$, the osculating cubic at $A_{2}$, which also passes through the associated intersection $A_{1}$ :

$$
Q_{2}: \quad\left(x_{3}+2 x_{2}\right)\left(x_{1}-x_{3}\right)^{2}+\lambda^{2}\left(3 x_{3}-2 x_{2}\right) x_{1}{ }^{2}=0 .
$$

Since every point of the line $x_{3}=x_{1}+x_{2}$ is invariant under the involution, three of the intersections of $Q_{1}$ and $Q_{2}$ lie on it. Two more are at each of $A_{1}$ and $A_{2}$. The only fixed point of the involution not on $x_{3}=x_{1}+x_{2}$ is the point $(1,1,0)$, which lies on neither cubic, so the two remaining intersections of $Q_{1}$ and $Q_{2}$ are interchanged by the involution, and hence lie on a fixed line passing through $(1,1,0)$. Suffice it to say that if $\lambda$ is real they may be shown to be conjugate imaginary.

Similarly, the cubics $Q_{3}$ and $Q_{4}$, osculating cubics at $A_{3}$ and $A_{4}$, may be obtained. Three of the intersections of $Q_{1}$ or $Q_{2}$ with $Q_{3}$ or $Q_{4}$ lie on a line, while the other six lie on a conic.

Just one more property seems worthy of mention. The points $S_{1}$ and $S_{1}{ }^{\prime}$ on the line $x_{3}=2 x_{1}$ may be given by the relation

$$
\left[(1-\lambda) x_{2}+2 \lambda x_{1}\right]\left[(1+\lambda) x_{2}-2 \lambda x_{1}\right]=0,
$$

or $x_{2}{ }^{2}-\lambda^{2}\left(x_{2}-2 x_{1}\right)^{2}=0$. This same line meets $Q_{1}$ at $A_{2}$ and at the points given by $x_{2}{ }^{2}+\lambda^{2}\left(x_{2}-2 x_{1}\right)^{2}=0$. These two pairs of points separate one another harmonically, though the second pair is imaginary when $\lambda$ is real.

Dartmouth College 